\title{
Indicadores de Qualidade e Produtividade como Instrumento de Apoio à Decisão no Processo de Expedição de Veículos
}

\author{
Ana Luiza Mendonça de Araujo \\ Engenheira de Produção - EE/UFRJ \\ Rua Almir de Almeida, 23 - Rio de Janeiro - RJ. Cep. 22750-130 \\ e-mail: mendonca.al@pg.com
}

Palavras-chave: armazenagem, expedição, medidas, produtividade, qualidade Keywords: measurement, productivity, quality, shipping, warehousing

\section{RESUMO}

Desde 1990, o governo brasileiro vem incentivando a implementação de Programas de Qualidade e Produtividade. Neste contexto, torna-se cada vez mais importante o estudo do desenvolvimento de sistemas de medidas de qualidade e produtividade, uma vez que eles dão apoio à aplicação de tais programas.

Os sistemas de medidas devem entretanto considerar a influência de fatores externos que podem afetar o desempenho real alcançado, resultando em conclusões errôneas a respeito da performance do trabalho.

Este artigo apresenta a definição de um conjunto de medidas de desempenho para a atividade de carregamento de caminhðes em uma Fábrica de Lubrificantes. Esta atividade é uma das mais importantes para a função de armazenagem em qualquer empresa. Ao final, foi realizada uma análise visando mostrar a importância de se considerar fatores externos que podem influenciar a produtividade.

\section{ABSTRACT}

Since 1990, Brazilian Government has been promoting the implementation of Quality and Productivity improvement programs. In this environment, it is important to study the development of quality and productivity measurement, once it supports the application of these programs.

Far from being easy, productivity measurement must consider the influence of external factors which may affect the real productivity achieved, resulting in wrong considerations about labor performance.

In this paper, a set of performance measures for the loading activity in a Lubricant Factory has been defined. This activity is one of the most important for the warehousing function in any company. Finally, an analysis has been made to show the importance of considering externalfactors that may influence productivity. 


\section{PRODUÇÃO}

\section{1 - Introdução}

Este estudo foi motivado pela necessidade de uma empresa reagir a um aumento da demanda, garantindo capacidade de atendimento, sem reduzir o nível de serviço prestado aos clientes. Uma das formas de se atingir tal meta é através da redução de ineficiências internas dos processos. É preciso compreender, entretanto, que para se obter ganhos na realização de uma determinada atividade, é importante monitorá-la tornando possivel a mensuração dos sucessos de cada modificação efetivada, bem como a garantia da previsibilidade $e$ da consistência do processo.

Um sistema de monitoramento permite que o processo permaneça sob controle ao longo do tempo, desde que ocorrências fora dos padrões estabelecidos sejam investigadas e tenham suas causas identificadas e eliminadas. Desta forma é possivel aprender com os problemas que surgem, evitando sua reincidência. Além disso, o sistema torna-se capaz de fomentar aumentos de produtividade através de metas justas e factíveis.

No Brasil, o governo tem incentivado a implementação de programas de melhoria da qualidade e produtividade nas empresas. A inserção do país no contexto das economias mais desenvolvidas é função da capacidade de modernização da indústria, em busca de maior eficiência. A modernização industrial requer a adoção de novos métodos de gerenciamento e gestão na empresa, bem como depende da capacidade de incorporação de novas tecnologias de produto e processo nas atividades desempenhadas.

$\mathrm{Na}$ ordem dessas considerações, os grandes desafios estão na busca da racionalização, da modernização e da competitividade, para a qual são indispensáveis a Qualidade e a Produtividade.

A competição internacional em bases tecnológicas, cenário que se apresenta ao país, será marcada fortemente pela relação entre os novos blocos econômicos, eliminando-se as tradicionais vantagens comparativas baseadas no uso de fatores de produção abundantes e baratos. Tal contexto estimulou o governo brasileiro a propor o Programa Brasileiro de Qualidade e Produtividade - PBQP, com o objetivo de estabelecer um conjunto ordenado de ações indutoras da modernização industrial e tecnológica, contribuindo para a retomada do desenvolvimento econômico e social.

Os projetos de alcance apenas interno às empresas, entretanto, não fazem parte do escopo do $\mathrm{PBQP}$, mas são indispensáveis para o alcance dos objetivos estabelecidos pelo programa. Neste contexto, torna-se importante estudar o desenvolvimento de sistemas de medição da qualidade e da produtividade uma vez que eles fazem parte e servem de auxílio ao desenvolvimento de programas de aprimoramento. 
Entretanto, a medição da produtividade não é uma coisa trivial pois deve considerar a influência de fatores conjunturais que podem afetar a produtividade alcançada. Muitas vezes pode-se estar monitorando um processo através do acompanhamento de medidas de produtividade da mão-de-obra ao longo do tempo e interpretá-las erroneamente por não se considerar fatores conjunturais. A influência desses fatores pode causar a redução da produtividade alcançada sem que a qualidade do trabalho dos operadores tenha sido reduzida.

A medição da qualidade, por outro lado, também possui fatores dificultadores pois depende muitas vezes do feedback fornecido pelos clientes. As empresas precisam não só de um sistema de medição mas também de um sistema de informação de escopo interno e externo que seja compatível com o monitoramento das atividades.

Neste estudo, foi definido um conjunto de medidas de qualidade e produtividade para a atividade de carregamento de caminhões de uma fábrica de lubrificantes, uma das mais importantes atividades do setor de armazenagem. $\mathrm{O}$ desempenho da atividade de carregamento não traz impacto apenas para a fábrica de lubrificantes, mas também para as empresas transportadoras que lhe prestam serviço, visto que é uma atividade que está intimamente ligada ao tempo de permanência dos caminhões na fábrica. A redução desse tempo através de melhorias de produtividade e qualidade não só reduz o tempo de entrega dos produtos aos clientes como também traz ganhos para as transportadoras, uma vez que o tempo parado para carga de produtos é responsável por uma grande parcela dos custos de transporte. Isso é particularmente relevante em rotas pequenas, nas quais o tempo para carga $e$ descarga é relativamente grande em comparação com o tempo de viagem.

Além disso, o tempo de carregamento possui influência no tempo de ciclo do pedido, pois representa uma das etapas ocorridas entre a colocação do pedido e a entrega dos produtos ao cliente. O tempo do ciclo do pedido e sua variabilidade são importantes dimensões do serviço ao cliente. Daí a relevância que deve ser atribuída ao monitoramento de todas as atividades que o compõem. Quanto mais adequado for o sistema de monitoramento e controle de tais atividades, mais consistente será o tempo de ciclo e maiores níveis de serviço poderão ser oferecidos aos clientes.

\section{2 - Revisão Bibliográfica}

Medidas são observações quantificadas de algum aspecto ou atributo de um processo, produto ou projeto. Elas aumentam nossa capacidade de compreender coisas inacessíveis às nossas habilidade e inteligência naturais. Desta forma, um sistema de medição pode ser um instrumento muito útil para a tomada de decisões.

De acordo com Ward (1996), medidas selecionadas adequadamente fornecem a 
base necessária para a atuação em um processo, uma vez que apoiam análises eficazes das atividades monitoradas. "Elas são essenciais para qualquer esforço direcionado à melhoria da qualidade. Não se pode controlar aquilo que não se pode medir".

Para se sustentar o aprimoramento contínuo, todas as atividades desempenhadas em uma organização deveriam ser medidas. $\mathrm{O}$ monitoramento é a base para o controle das atividades. Além disso as medidas podem funcionar como instrumento de motivação. Segundo Moreira (1991), "a mera existência de estimativas de produtividade pode servir de estimulo para que funcionários passem a raciocinar em termos de eficiência e preocupar-se com elas".

Devido à grande importância dos sistemas de medidas para as empresas, é fundamental selecionar métricas que expressem com fidelidade aquilo que está se tentando mensurar.

De acordo com Byrne (1991), as empresas utilizam sistemas de medição de qualidade e produtividade para: facilitar a comunicação; identificar áreas que precisam ser melhoradas; obter dados que ajudam a entender problemas; avaliar alternativas; acompanhar progressos na direção de metas; quantificar e informar resultados de melhorias; fornecer feedback do desempenho obtido para os clientes; além de, é claro, alimentar as análises estatisticas.
Para decidir o que deve ser medido, uma empresa deve fazer quatro perguntas: "O que se está tentando realizar?"; "O que representa uma boa medida disso?"; "Qual fórmula matemática calcula isso?"; "Quais são as fontes de dados?'. Seguindo essa seqüência lógica, pode-se evitar perder-se no meio de dados excessivos, cuja grande quantidade limita a capacidade de análise. Ao mesmo tempo, pode-se assegurar que as medidas estão atingindo $o$ alvo correto.

\section{1 - Medição da Qualidade do} Serviço Logístico

As medidas de qualidade do serviço representam uma estimativa da percepção dos clientes a respeito do serviço prestado por uma empresa. Em vista disso, para que tais métricas sejam eficazes é necessário, em primeiro lugar, compreender as necessidades e expectativas dos clientes.

Além disso, é cada vez maior o número de clientes que vêm implementando seu próprio sistema de monitoramento da qualidade do serviço para avaliar seus fornecedores. A compatibilidade, em termos de expectativas, entre um sistema de monitoramento interno e o dos clientes é de fundamental importância.

Em um estudo realizado em $1987 \mathrm{em}$ empresas dos Estados Unidos, conforme mostrado por Stock e Lambert (1992), concluiu-se que atributos do produto e do 
serviço ao cliente contribuem mais fortemente para garantia de mercado de uma empresa de manufatura do que outros atributos do marketing mix tais como preço e promoção.

Os resultados da pesquisa mostraram que o serviço logístico é um componente necessário do marketing mix que pode fornecer às empresas oportunidades significativas para obtenção de vantagem competitiva. "O ponto de ligação entre a logística e o gerenciamento/planejamento estratégico é o serviço ao cliente." (1992)

De acordo com Stock e Lambert (1992), é nesta área que a logística pode representar o maior impacto na qualidade do serviço. Neste contexto, é importante ressaltar a relevância das atividades de armazenagem de produtos e da garantia da qualidade nestas atividades.

Para se obter vantagens através da qualidade do serviço logístico, é necessário que o fornecedor atenda às expectativas dos clientes nos atributos mais importantes do serviço. Stock e Lambert (1992) colocam que o primeiro passo para atingir esse objetivo é desenvolver estratégias para melhorar o desempenho nas atividades que se relacionam com tais atributos. "Para isso, é extremamente importante medir o desempenho dos vários componentes do serviço logístico. É impossível gerenciar aquilo que não é monitorado. Além disso, uma empresa precisa ser capaz de medir os resultados de suas ações." (1992)
De acordo com Byrne (1991), as medidas de qualidade do serviço devem englobar dois tipos de medidas: as "essenciais" e as de "valor agregado". As "essenciais" - entrega sem atraso; acurácia da documentação; número de avarias etc. - são geradas internamente. As de "valor agregado" dependem do feedback dos clientes a respeito de como os serviços prestados estão em relação a suas expectativas.

A definição de um conjunto de métricas para a qualidade de levar em conta que resultados numéricos não expressam as razões das falhas ou sucessos. Por isso mais informações são necessárias para que as medidas sejam consideradas robustas.

Com essa finalidade Byrne (1991) lista três tipos de medidas a serem utilizadas para monitorar a qualidade do serviço:

- Medidas de Resultado: "Como estamos?" - fornecem um retrato do desempenho obtido em determinado atributo. Podem ser usadas para identificar tendências e comparar o desempenho alcançado com as metas pré-estabelecidas.

- Medidas de Diagnóstico: "Por quê?" - fornecem os motivos e as fontes do desempenho obtido.

- Medidas de Impacto: "A que custo?" - fornecem o custo do desempenho obtido. 


\section{2 - Medição da Produtividade}

Medir a produtividade de uma atividade logistica significa medir a eficiência com que o processo logistico atende aos anseios dos clientes. Cabe aqui fornecer uma definição clara para "produtividade". O conceito é simples e pode ser expresso, como o faz Byrne (1991), como sendo a razão entre o output real produzido e o input real consumido, onde:

- Output é uma medida do trabalho realizado por uma atividade (por exemplo, número de caminhões carregados, quilogramas carregados, etc.).

- Input é uma medida do recurso consumido para realizar o trabalho (por exemplo, homem-hora ou horas de operação de empilhadeira).

Esquematicamente pode-se colocar, então, que:

$$
\text { Produtividade }=\frac{\text { Output Produzido }}{\text { Input } \text { Consumido }}
$$

Duas questões, colocadas por Byrne (1991), devem ser cuidadosamente verificadas por aqueles que planejam desenvolver sistemas de medição da produtividade:

(1) - O output produzido possui valor; não se trata necessariamente de "esforço dispendido".

(2) - Tanto o output produzido quanto o input consumido não podem ser afetados por mudanças monetárias.

O objetivo principal de se estabelecer um sistema de medição de produtividade para uma determinada atividade é prover a informação necessária para melhorar a produtividade, a alocação de pessoas e a performance do trabalho. Torna-se importante monitorar os resultados correntes, compará-los com valores históricos, medir o desempenho dos operadores, monitorar os progressos obtidos e até mesmo auxiliar o processo de avaliação de empregados.

Convém ressaltar a diferença entre desempenho (performance) e produtividade (conforme apresentado anteriormente, output contra input). Desempenho é uma medida do nível individual de esforço e capacidade, é a razão entre o output real e o output padrão.

A definição de padrões de desempenho é bastante útil para a manutenção do nível desejado de produtividade. Esta definição pode ser feita a partir de diferentes técnicas, como por exemplo, estudo de tempos e movimentos, cálculo de estimativas ou metas de produtividade ou médias históricas.

Na verdade, como coloca Moreira (1991), pode ser enganoso o uso da palavra produtividade num sentido genérico. Não existe uma, mas sim várias medidas da produtividade. Para cada função, um grande número de elementos de trabalho podem ser avaliados, cada um 
com sua unidade de medida própria. A produtividade designa uma familia de relações entre produção e insumos, alterando-se a particular relação dependendo de quantos e quais insumos serão levados em conta. O uso de uma relação, ou seja, de uma medida de produtividade em detrimento de outra estará condicionado em princípio aos objetivos pretendidos com a medida, mas também a inevitáveis problemas práticos que forçam geralmente a simplificações.

De acordo com Byrne (1991), a produtividade pode ser aumentada de três formas: reengenharia do processo $\mathrm{em} \mathrm{si}$, melhoria da utilização dos recursos e aumento do desempenho (performance) através de metas ou outros incentivos.

Em qualquer ramo da indústria, ser melhor do que os concorrentes em um atributo de grande valor para os clientes é uma fonte de vantagem competitiva e garantia de mercado. O monitoramento e controle da produtividade no carregamento de caminhões auxilia na obtenção de ciclos de pedido menos variáveis. Porém, mais do que isso, é importante ressaltar que, no Brasil, as atividades de carregamento costumam ser notadamente ineficientes e demoradas. Sendo assim, obter-se maior produtividade e controle no carregamento pode ser o primeiro passo para negociações acerca do preço do frete vantajosas tanto para o embarcador de produtos quanto para os transportadores.

A importância do monitoramento e controle das atividades de armazenagem fica muito clara quando se chama atenção para o impacto dessas atividades na satisfação do cliente. De acordo com uma pesquisa realizada nos EUA ("The 199293 Logistics Management and Technology Benchmarking Survey"), muitas empresas já utilizam medidas de produtividade e qualidade na armazenagem, conforme mostra Muroff (1993). A pesquisa, um esforço coordenado de C. S. Report Inc. e KPMG Peak Marwick, estudou 110 empresas de varejo, atacado e manufatura. Segundo Muroff (1993), quase todas as empresas pesquisadas utilizam pelo menos uma medida de qualidade e uma de produtividade para atividades de armazenagem. Quanto maior a aceitação e a implementação de sistemas de monitoramento da produtividade na armazenagem, maior será o desempenho alcançado.

\section{3 - A Empresa}

A fábrica de lubrificantes onde foi realizado este estudo possui um faturamento de cerca de 20 milhões de dólares anuais e sua participação no mercado brasileiro é de $7,9 \%$.

A partir de 1993, a empresa passou por uma fase de grandes mudanças que culminaram num aumento do volume de produção de praticamente $100 \%$. Tais mudanças representaram ainda uma reviravolta cultural na empresa, que vinha de um longo período de poucos investimentos. Atualmente, a alta administração tem grande interesse de que a empresa consolide-se cada vez mais em seu mercado de atuação e dá grande 


\section{PRODUÇÃO}

importância ao processo logístico como fonte de vantagem competitiva.

Desta forma, projetos relacionados não só a modificações técnicas, mas também a mudanças no modo de gerenciar e nos instrumentos utilizados para isso, possuem grande relevância para a nova realidade da empresa.

\section{1 - A Armazenagem de Produtos Acabados}

A armazenagem de produtos acabados da fábrica de lubrificantes consiste, na verdade, de dois armazéns separados: um armazém para produtos embalados em baldes e tambores; e um armazém para produtos embalados em caixas de papelão (ver Fig. 1).

As atividades numeradas na Fig. 1 são: 1. Recebimento: retirada dos sistema FIFO ("first in, first out"), as produtos embalados através de uma esteira. Tais produtos são transportados por um pequeno trem que circula por trás dos armazéns, vindo do setor de Enchimento. Os produtos ficam empilhados por alguns instantes na área de recebimento, que é pequena.

2. "Put-away": uma empilhadeira retira os pallets da área de recebimento $\mathrm{e}$ os coloca na área de estocagem, que ocupa praticamente todo o armazém. Não existe local pré-estabelecido para cada tipo de produto.

3. Estocagem: os produtos são estocados em pallets. No armazém de caixas eles são guardados em prateleiras do tipo "drive-in", enquanto que no armazém de baldes os pallets ficam empilhados uns sobre os outros.

4. Seleção de Pedidos: seguindo o

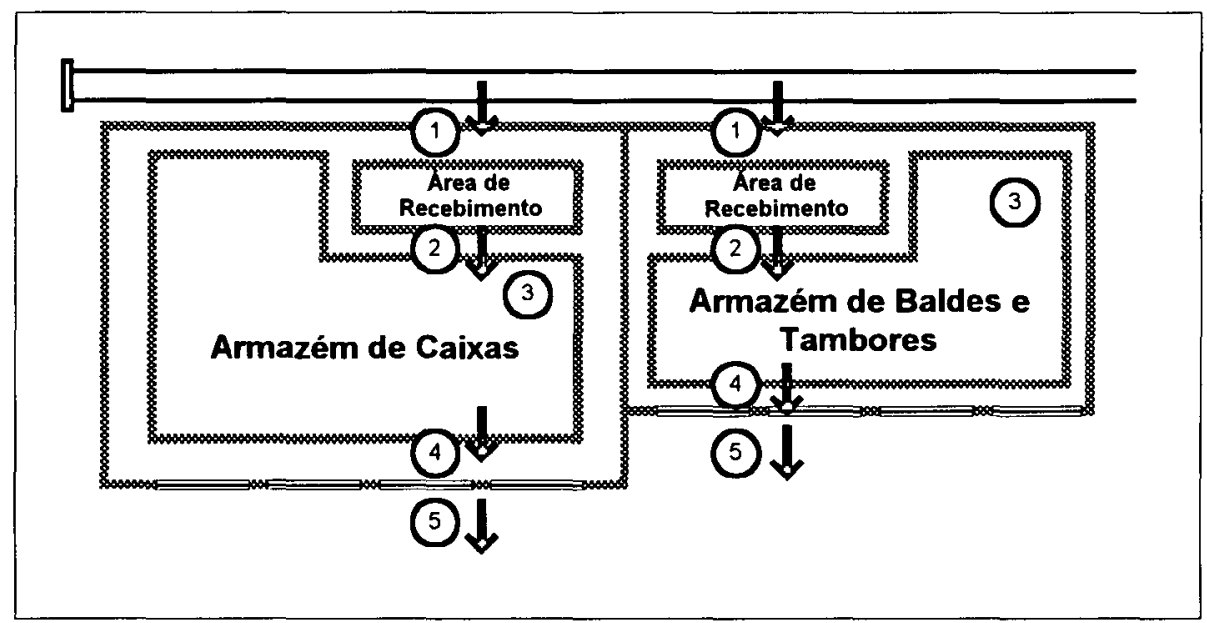

Fig. 1 - Armazenagem na Fábrica de Lubrificantes 
empilhadeiras retiram os produtos do seu local de estocagem e os levam para as docas de carregamento. Não existe prémontagem de carga.

5. Embarque/Carregamento: os pallets são colocados sobre a carroceria dos caminhões pelas empilhadeiras e os produtos são despaletizados e arrumados manualmente sobre o caminhão.

A documentação associada a essas operações são preparadas pelo setor administrativo responsável (Seção de Faturamento e Expedição). A descrição acima vale para os dois armazéns, já que a operação é semelhante em ambos os casos.

Cabe lembrar ainda que os dois armazéns da Fig. I encontram-se desnivelados, de forma que as empilhadeiras não podem circular entre eles. Sendo assim, caminhões que chegam para carregar os dois tipos de embalagem (a imensa maioria), encostam primeiro em uma doca do armazém de baldes e tambores e depois seguem para uma doca do armazém de caixas.

Em vista disso, a circulação de caminhões no pátio da empresa é uma questão crítica. $O$ espaço livre para manobras não é muito grande, por isso, quanto menor o tempo de permanência dos caminhões no pátio da fábrica, mais fácil se torna a organização interna do tráfego de veículos.

Com a duplicação da demanda e da produção de lubrificantes, o setor de armazenagem ficou sobrecarregado. $\mathrm{O}$ controle dos níveis de produtividade do carregamento dos caminhões e a garantia da qualidade nessa atividade poderiam ser, portanto, instrumentos úteis para que o ciclo de pedidos não fosse prejudicado.

Além disso, através do conhecimento prévio do tempo necessário para carregar determinado veículo, torna-se mais fácil a programação de transportes, podendose determinar junto às transportadoras horários para chegada de veículos, minimizando 0 problemático congestionamento no pátio e utilizando racionalmente a capacidade do setor.

\section{2 - A Atividade de Carregamento de Caminhões}

Como pôde ser visto no item anterior, uma das mais importantes atividades do setor de armazenagem da fábrica é o carregamento de caminhões.

O caminhão, truck ou carreta, após estar pronto para carregar, encosta em uma doca do armazém de latas e baldes. A carga é movimentada por duas empilhadeiras e despaletizada manualmente dentro do caminhão. Após o término do carregamento no armazém de baldes, o caminhão deixa a doca e segue para outro o armazém, onde é realizado o carregamento de caixas. Uma vez na doca, a carga é colocada no caminhão da mesma forma que no armazém de baldes. Cabe lembrar que um truck tem capacidade de até $15.000 \mathrm{~kg}$ e uma carreta carrega até $30.000 \mathrm{~kg}$. 


\section{4 - Proposta para 0 Monitoramento e Controle da Qualidade do Serviço}

De acordo com os conceitos anteriormente explorados sobre a medição da qualidade do serviço logístico, foram definidas algumas medidas adequadas ao monitoramento da qualidade do serviço para a atividade de carregamento e embarque de produtos na fábrica de lubrificantes. As métricas expostas a seguir podem ser usadas para avaliar a qualidade do processo e englobam medidas de Resultado (quais são os requisitos?), de Diagnóstico (porque os requisitos não estão sendo satisfeitos?) e de Impacto (efeito do não cumprimento dos requisitos).

\section{Indices de Resultado}

- Percentual de unidades embarcadas corretamente (por veículo): \%UEC - A implantação desta medida depende de um constante feedback por parte dos clientes ou transportadores, para alimentação do sistema de monitoramento. Ao término das entregas realizadas por cada veículo, o transportador deve registrar de forma apropriada o número de unidades embarcadas erroneamente. Essa informação, ao ser enviada para a fábrica, alimentará o sistema que calculará o percentual de unidades embarcadas corretamente:
$\% \mathrm{UEC}=$ Total de unidades embarcadas no veículo - Total de unidades erradas $x$ $100 /$ Total de unidades embarcadas no veículo

\section{- Percentual de carregamentos} realizados corretamente (por mês): \%CRC - Da mesma forma que o indice anterior, esta medida depende do feedback dos transportadores (ou clientes). Este índice difere do anterior pois considera um carregamento "incorreto" se houver produtos errados no mesmo, independente da quantidade de produtos errados. O sistema de monitoramento calculará esse indice da seguinte forma:

$\%$ CRC $=$ Qtde. de carregamentos no mês - Qtde. de carregamentos c/ erro no mês X 100/Qtde. de carregamentos no mês

\section{Índices de Diagnóstico}

- Percentuais de carregamentos incorretos, segregados por motivos do erro (por mês):

* Lista de produtos (romaneio) incorreta.

* Seleção de produtos incorreta.

* Substituição de produto não autorizada.

* Produto com avarias.

A implementação deste indice depende da investigação das causas dos erros no carregamento. No início da implantação do sistema de monitoramento, enquanto a atividade 
ainda está fora de controle, isso poderá ser muito freqüente e cansativo. Porém, uma vez controlada a atividade e eliminadas as causa comuns, apenas ocorrências especiais (fora dos limites estabelecidos) entrarão no cálculo deste índice. Esse processo é dinâmico e as modificações na atividade visando eliminar causas de erros, provocarão a necessidade de constantes mudanças na lista de "motivos" apresentada acima.

- Percentuais de carregamentos realizados com produtividade inferior ao limite mínimo estabelecido, segregados por motivos (por mês):

* Seleção de produtos incorreta.

* Falta de empilhadeira.

* Falta de ajudantes.

* Lista de produtos (romaneio) incorreta.

* Falta de estoque.

\section{Índice de Impacto}

- Custo dos erros ou atrasos no carregamento (por mês):

* Frete pago para retorno de produtos.

* Custo do processamento interno da devolução de produtos.

* Estimativa de vendas perdidas devido à insatisfação do cliente.

Este último "item de custo" é muitas vezes subjetivo e depende da troca de informação com vendedores, que têm maior contato com clientes. Entretanto, possui fundamental importância, e é um dos mais representativos custos da má qualidade.

\section{5 - Proposta para o Monitoramento e Controle da Produtividade}

A partir de informações obtidas acerca da operação, foram selecionadas algumas medidas de produtividade adequadas às atividades de embarque. Essas medidas classificam-se nos seguintes grupos: trabalho, instalações e equipamentos.

\section{Índices de Trabalho}

- Taxa de carregamento em quilogramas por hora: representa o peso médio (em $\mathrm{Kg}$ ) carregado em cada caminhão no intervalo de uma hora. $\mathrm{O}$ cálculo é feito dividindo-se o peso total carregado em cada caminhão pela diferença entre o horário final e o horário inicial de carregamento deste caminhão. Uma variação dessa medida é o cálculo da taxa de carregamento em quilogramas por homem-hora.

- Veículos carregados por hora (em carretas/hora ou trucks/hora): representa a fração de veículo (truck ou carreta) que é carregada em uma hora. É calculado dividindo-se a taxa de carregamento pela carga média do caminhão considerado.

- Performance relativa entre diferentes tipos de caminhão: representa a fração de um caminhão carregada em tempo correspondente ao tempo de 


\section{PRODUÇÃO}

carregamento total de outro caminhão de tipo diferente. Esse índice mostra, por exemplo, que o tempo necessário para carregar 0,60 carreta é o mesmo necessário para carregar 1 truck. O cálculo é a razão entre as medidas de veiculos carregados por hora de diferentes tipos de caminhão.

\section{Índices de Instalações}

- Produtividade diária por doca (em $\mathrm{Kg}$ /doca.dia): representa a taxa diária de carregamento numa doca, ou seja, quantos quilogramas foram movimentados em cada doca em um dia. Seu cálculo é feito dividindo-se o total carregado em um dia (em $\mathrm{Kg}$ ) pelo número de docas em operação neste dia.

- Giro de doca (em Veículo/ doca.dia): representa o número médio de veículos que encostam para carregar em cada doca em um dia. É calculado dividindo-se o número de veículos carregados em um dia pelo número de docas em operação neste dia. Esse índice pode ainda ser expresso em carretas/ doca.dia ou trucks/doca.dia.

\section{Índice de Equipamentos}

- Produtividade das empilhadeiras (kg/hora.empilhadeiras): representa o peso (em $\mathrm{Kg}$ ) movimentado por cada empilhadeira no intervalo de uma hora. É calculado dividindo-se o total diário carregado (em $\mathrm{Kg}$ ) pelo produto entre o número de empilhadeiras e o total de horas de utilização por dia.

\section{6 - Implementação das Medidas de Produtividade}

\section{1 - Experimentação Inicial dos Índices}

Para os Índices de Trabalho, Instalações e Equipamentos selecionados, foram obtidos os valores médios abaixo, a partir de uma amostra de 127 veículos (4 dias). Nesta amostra, $58 \%$ dos veículos eram carretas e $42 \%$ eram trucks.

\section{Índices de Trabalho}

$\begin{array}{ll}\text { Taxa de carregamento } & \\ \text { Para veiculos em geral } & 3818,30 \mathrm{Kg} / \mathrm{h} \\ \text { Para trucks } & 2942,51 \mathrm{Kg} / \mathrm{h} \\ \text { Para cametas } & 4496,32 \mathrm{~kg} / \mathrm{h} \\ \text { Velculos carregados por hora } & \\ \text { Para veículos em geral } & 0,21 \text { vefculos/hora } \\ \text { Para tuclss } & 0,26 \text { trucls } / \mathrm{hora} \\ \text { Para carretas } & 0,19 \text { carretas/hora }\end{array}$

Performance Relativa

TCruck $=0,70$ TCearmet

onde TC = tempo de carregamento (médio da amostra)

\section{Índices de Instalações}

Produtividade diária por doca

Para veículos em geral

$40267,68 \mathrm{Kg} /$ doca.dia

Giro de Doca

Para vefculos em geral

2,12 vé́culos/doca.dia

Para trucks

0,88 trucles/doca.dia

Para carretas

1,23 carretas/doca:dia 


\section{PRODUÇÃO}

\section{Taxa de carregamento}

Produtividade das Empilhadeiras

$8628.79 \mathrm{Kg} /$ hora.empilhadeira

Como já foi citado, os valores apresentados acima representam apenas os valores médios, obtidos num pequeno intervalo de tempo (apenas quatro dias), para as medidas sugeridas. Para um aproveitamento eficaz do sistema de medição de produtividade, faz-se necessário o acompanhamento dos valores dos índices ao longo do tempo, comparando-os com valores históricos ou metas a serem atingidas. A comparação das medidas antes e depois da implementação de alguma modificação no sistema de operação pode dar uma visão compreensivel da eficácia dessa mudança.
Para exemplificar e mostrar como a visualização gráfica facilita a análise das medidas, é apresentado abaixo o gráfico do indice de trabalho Taxa de Carregamento. Cada ponto representa a média diária no período de 17 de fevereiro a 01 de abril de 1995.

As médias obtidas para produtividade de trucks e carretas foram $4831.78 \mathrm{Kg} / \mathrm{h}$ e $6067.80 \mathrm{Kg} / \mathrm{h}$, respectivamente.

Através da Fig. 2 apresentada abaixo, pode-se notar que a produtividade do carregamento em carretas é quase sempre maior que em trucks, apesar deste último veículo apresentar aumentos de produtividade proporcionalmente maiores que os das carretas. De qualquer forma, como a carreta comporta em média o dobro do volume de carga que um truck,

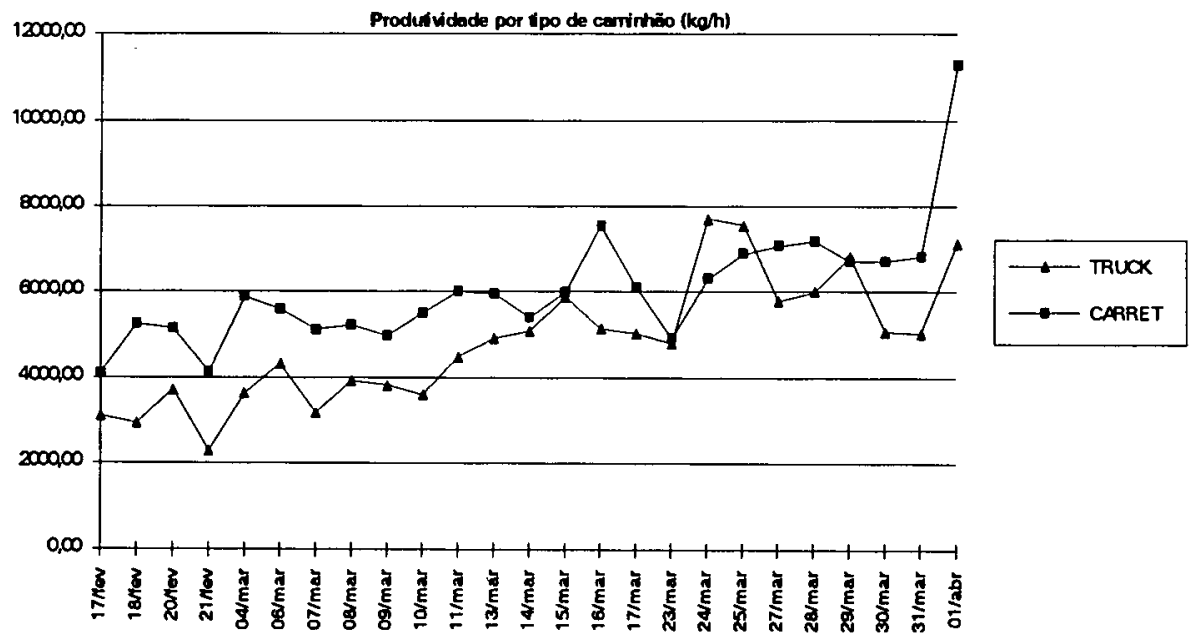

Fig. 2 - Gráfico Taxa de Carregamento (Trucks e Carretas) 
porém apresenta uma taxa de carregamento (produtividade) maior, concluimos que há um ganho de escala no carregamento, ou seja: apesar da carreta ser duas vezes maior que um truck, o tempo de embarque não é necessariamente o dobro. Este ganho pode ser facilmente explicitado pelo fato do valor médio obtido anteriormente para o indice Performance Relativa entre carretas e trucks ter sido igual a $70 \%$, ou seja, o tempo necessário para carregar um truck equivale ao tempo necessário para carregar $70 \%$ de uma carreta (em média), ao contrário dos esperados $50 \%$ se não houvesse ganhos de escala.

Sendo assim, ao longo da análise dos dados coletados e a partir de informações fornecidas pelas pessoas responsáveis pelo carregamento na fábrica de lubrificantes, ficou evidenciada uma interdependência entre alguns fatores que influenciam na produtividade do carregamento, como o tipo de caminhão (carreta ou truck) e o tipo de carga (fracionada ou unitizada). Decidiu-se analisar, então, se realmente existe influência de tais fatores na produtividade alcançada e, em caso afirmativo, com que magnitude eles influem na taxa de carregamento.
O objetivo seria eliminar interpretações errôneas acerca das variações dos valores da produtividade. Por exemplo, se o valor médio da produtividade da mão-de-obra de um mês for menor do que o observado no mês anterior, isso não significa necessariamente que a qualidade do trabalho dos operadores diminuiu. Pode ter havido maior ocorrência de caminhões com cargas fracionadas, ou o percentual de trucks pode ter sido consideravelmente maior do que o de carretas.

\section{2 - O Projeto de Experimentos}

O principal objetivo desta etapa foi obter informações sobre a sensibilidade do sistema a variações em dois fatores, que pareciam influenciar mais fortemente seu desempenho. Esses fatores são o tipo de caminhão (carreta ou truck) e o tipo de carga (fracionada ou unitizada).

Em primeiro lugar, decidiu-se verificar se realmente existia influência desses fatores na produtividade alcançada. Para isso foi utilizada uma amostra de 668 carregamentos, coletada ao longo dos meses de fevereiro, março e abril de 1995. Essa amostra foi subdividida em quatro conjuntos de dados da seguinte forma:

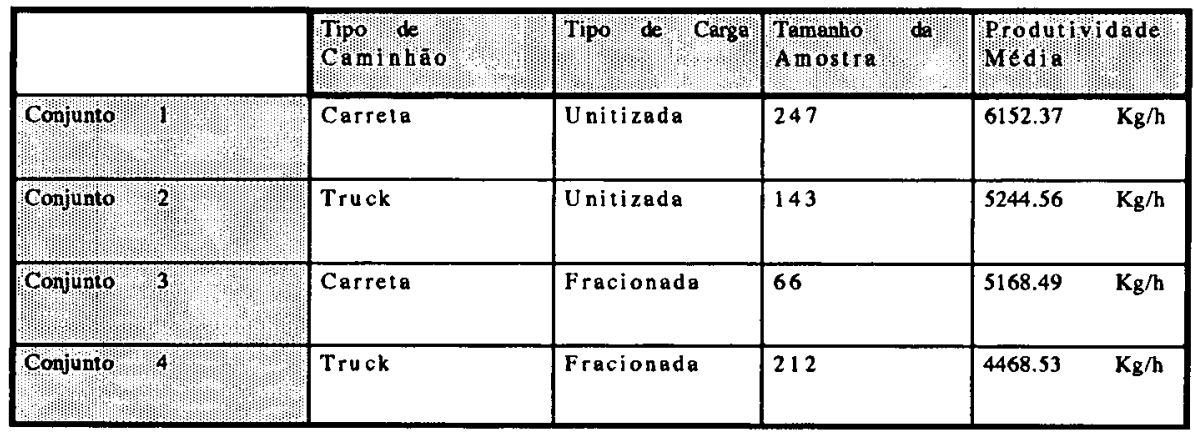

Tabela 1 - Amostra de Carregamentos 
A produtividade média obtida para 0 Conunto 4 foi, conforme esperado, menor do que a média obtida para o Conлunto 1 .

Com o objetivo de verificar se as produtividades médias de cada um dos quatro conjuntos eram significativamente diferentes, foi realizada uma Análise de Variância (ANOVA) com as amostras. A partir do resultado dessa análise (apresentado no próximo item) pôde-se rejeitar a hipótese nula de igualdade das quatro médias, ou seja, a produtividade é maior no caso de carretas e de cargas unitizadas.

O próximo passo consistiu em identificar a importância relativa dos fatores sobre a performance do sistema. Com isso seria possivel determinar qual deles afeta mais a produtividade do carregamento. Além disso, seria A nova

RESUMO interessante verificar se existe interação significativa entre o par de fatores. Foi realizado então, um projeto fatorial simples mediante auxílio de software estatístico (SPSS).

O projeto fatorial permite isolar os efeitos principal e secundário dos fatores em questão. (1992) O efeito principal indica a importância de um dado fator (tipo de carga, por exemplo) para o desempenho do sistema, considerando-se o conjunto das configurações possiveis. $O$ efeito secundário indica a possível interação entre os dois fatores. Os resultados estão apresentados no próximo item.

\subsection{2 - Apresentação dos Resultados}

Na Tabela 2 abaixo está apresentado o resumo das estatísticas obtidas após os cálculos da Análise de Variância (ANOVA).

\begin{tabular}{|c|c|c|c|c|}
\hline Grupo & $\begin{array}{l}\text { No de } \\
\text { observacos }\end{array}$ & soma & Média & Verrancia \\
\hline CarretaUnitizada & 247 & 1519635.63 & 6152.37096 & 8688128.48 \\
\hline TruekUnitizada & 143 & 749972.226 & 5244.56102 & 14613653.9 \\
\hline Carreta Fracionada & 66 & 341120.4 & 5168.49091 & 3681534.8 \\
\hline TruckFracionada & 212 & 947330.334 & 4468.53931 & 6625091.36 \\
\hline
\end{tabular}

\begin{tabular}{|c|c|c|c|c|c|c|}
\hline Fompda & 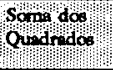 & $\begin{array}{l}\text { Broundo } \\
\text { libordade }\end{array}$ & 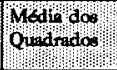 & F & forp & Frrtio \\
\hline Entre grupos & 327129900 & 3 & 109043300 & 12.3777004 & $6.9305 \mathrm{E}-08$ & 2.61832156 \\
\hline $\begin{array}{l}\text { Deniro dos } \\
\text { grupos }\end{array}$ & 5849612496 & 664 & 8809657.37 & & & \\
\hline Total & 6176742396 & 667 & & & & \\
\hline
\end{tabular}

Tabela 2 - Resumo das Estatísticas Obtidas no Cálculo da ANOVA 
Decompondo a variação total da produtividade do carregamento nos componentes tipo de carga e tipo de caminhão, pode-se testar se existe diferença discernivel entre os quatro conjuntos. Em qualquer dos testes será levada em conta a influência estranha do outro fator.

Se a hipótese nula ( $\mathrm{H} 0)$ de igualdade das médias for verdadeira, a razão entre a variância explicada e a variância não explicada deve ter distribuição $F$.

Desta forma, para três graus de liberdade entre os grupos e 664 graus de liberdade dentro dos grupos, tem-se que o valor crítico de $F$ é 2,61 contra 12,37 da razão entre as variâncias. Conseqüentemente conclui-se que, ao nível de $5 \%$, há diferença discernivel entre as quatro médias.

No projeto fatorial, onde novamente a variável dependente foi a produtividade (taxa de carregamento) e os fatores foram o tipo de carga e o tipo de caminhão obteve-se os seguintes resultados (tabela abaixo):

Pôde-se concluir, portanto, que existem efeitos principais significativos para os dois fatores (significância de $\mathrm{F}$ igual a 0,001 para Tipo de Carga e 0,002 para Tipo de Caminhão) mas que não existe interação entre esses fatores

\begin{tabular}{|c|c|c|c|c|c|}
\hline 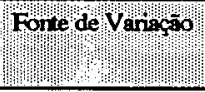 & 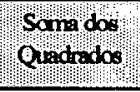 & Grans dẹ & 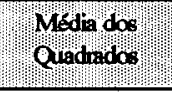 & 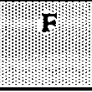 & 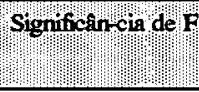 \\
\hline \multicolumn{6}{|l|}{ Efeitos Principais } \\
\hline & 323719072 & 2 & 161859536199 & 18373 & .000 \\
\hline Tipo de Carga & 100202021 & 1 & 100202020.848 & 11.374 & .001 \\
\hline Tịo de Caminhão & 836264411 & 1 & 83626410.856 & 9.493 & .002 \\
\hline 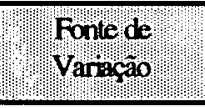 & Somp dos! & Graw de & $\begin{array}{l}\text { Media dos } \\
\text { Qcadrados }\end{array}$ & $\mathrm{f}$ & Significan cia de \\
\hline \multicolumn{6}{|l|}{$\begin{array}{l}\text { Efeitos } \\
\text { Secundários }\end{array}$} \\
\hline Tipo de Carga & 1397779 & 1 & 1397778855 & 159 & 691 \\
\hline $\begin{array}{l}\text { Tpo de } \\
\text { Caminhão }\end{array}$ & $139 m 79$ & 1 & $139 / 778855$ & 159 & 691 \\
\hline
\end{tabular}

Tabela 3 - Resultados do Projeto Fatorial 
(significância de $\mathrm{F}$ igual a 0,691 para o efeito secundário).

Uma vez que não existe interação relevante entre os dois fatores, o modelo pode ser considerado aditivo. Desta forma, um meio de se estimar o efeito de cada fator é calcular a regressão linear múltipla da produtividade como variável dependente, função do tipo de carga e do tipo de caminhão. Cabe ressaltar que os dois fatores foram tratados como variáveis do tipo 0 ou 1 , ou seja, valores intermediários para o tamanho do caminhão e para o nivel de unitização da carga não foram considerados.

Obs.: 1 Carga fracionada $=0$; Carga unitizada $=1$.

2 Truck $=0 ;$ Carreta $=1$.

A estatística $T$ apresenta, para os dois coeficientes das variáveis independentes, valores superiores a 1,96. Sendo assim, os coeficientes apresentam significância estatística para um intervalo de confiança de $95 \%$, podendo ser considerados boas estimativas para o efeito dos fatores na produtividade.

A equação obtida pela regressão é a seguinte:

Produtividade $=854,77 *$ Tipo de Carga $+833,56 *$ Tipo de Caminhão + 4436,82

Finalmente, pode-se concluir que o carregamento de carga unitizada em um determinado caminhão provoca um aumento médio na produtividade de $854,77 \mathrm{Kg} / \mathrm{h}$ em relação ao carregamento de carga fracionada. Já o efeito de carregar-se em carreta representa um aumento médio de $833,56 \mathrm{Kg} / \mathrm{h}$ na produtividade, comparando-se ao carregamento em truck. S e compararmos a variação da média das amostras com a média global ( $\mathrm{M}=$ $5326,44 \mathrm{Kg} / \mathrm{h}$ ) teremos os seguintes resultados, para as configurações mais favorável e menos favorável:

\begin{tabular}{|c|c|c|c|c|c|}
\hline 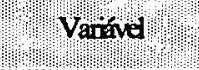 & Goeficuter & Enopadrio & Bun & Estarsoin T & $\mathrm{Sg} \cdot \mathrm{deT}$ \\
\hline Tpode Carga & 854766573 & 252978985 & 138559 & 3379 & 8 \\
\hline Tpo de Caminhão & 833560033 & 24989259 & 136790 & 3336 & 9 \\
\hline Constante & 44368119381 & 187534017 & & 23699 & 0 \\
\hline
\end{tabular}

Tabela 4 - Resultados da Regressão Linear Múltipla 
Pode-se perceber que, se 0 carregamento fosse executado apenas em carretas com carga unitizada, a produtividade seria $15,5 \%$ maior do que a média atual, enquanto que se fosse executado somente em trucks com carga fracionada, sua produtividade cairia 16,1 $\%$ em relação à média. Esta é uma outra forma de visualizar a influência desses fatores na produtividade do carregamento.

\section{9 - Conclusões}

Conforme pôde ser constatado, a produtividade do carregamento de caminhões na fábrica de lubrificantes é realmente influenciada pelos fatores tipo de carga e tipo de caminhão, embora possam existir outros fatores não considerados neste estudo que também exercem influência sobre a produtividade. Essa conclusão pode ser útil, por exemplo, na definição de entregas a serem realizadas, que pode ser feita considerando a influência dos dois fatores considerados e os custos relacionados às variações de produtividade do carregamento.
Os resultados aqui apresentados mostram o potencial existente na implementação de um sistema de medição de desempenho. Ainda que a influência dos fatores carga e caminhão na produtividade alcançada possa parecer óbvia para as pessoas que operam no diaa-dia da movimentação de materiais da fábrica, aqui ela pôde ser quantificada, podendo-se a partir disso estimar possíveis ganhos a serem obtidos com o controle dos fatores considerados.

Da mesma forma, o acompanhamento dos valores coletados para os outros indices sugeridos, e não apenas a medida taxa de carregamento, poderá levar ao surgimento de outras análises ou à percepção de outras influências ainda não detectadas que poderão trazer ganhos expressivos ao setor de carregamento de baldes e caixas.

Quanto à formalização de um sistema de medição de qualidade e produtividade, muito ainda pode ser acrescentado, no sentido de implementar o sistema aqui proposto de coleta, tratamento e análise

\begin{tabular}{|c|c|c|c|}
\hline प्र & 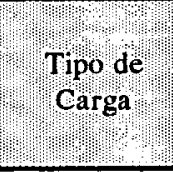 & 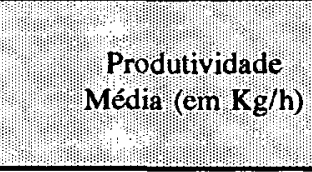 & 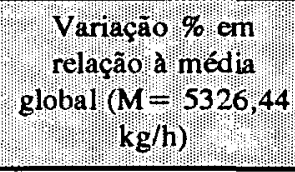 \\
\hline Carreta & Unitizada & 615237096 & $+15,5 \%$ (melhor) \\
\hline Truck & Fracionada & 446853931 & $-16,1 \%$ (pior) \\
\hline
\end{tabular}

Tabela 5 - Influências 


\section{PRODUÇÃO}

de dados e de definir conjuntos de medidas adequados a outras atividades logísticas da fábrica, para as quais haja interesses específicos.

\section{0 - Referências Bibliográficas}

1. BYRNE, Patrick M.; MARKHAM, William J. Improving quality and productivity in the logistics process. Chicago: Council of Logistics Management, 1991.

2. FLEURY, Paulo F. Estrutura de produção e desempenho operacional: identificação de variáveis chave através de simulação. Rio de Janeiro: COPPEAD/ UFRJ, 1992.

3. MEASURING and improving productivity in physical distribution. Chicago: National Council of Physical Distribution Management, 1984. Apêndice B: Measuring and improving productivity in warehousing.

4. MOREIRA, Daniel A. Medida da produtividade na empresa moderna. São Paulo: Livraria Pioneira Editora, 1991.

5. MUROFF, Cindy. Warehouse productivity proves surprising. Distribution, v. 4, p. 76-78, Janeiro 1993.
6. STOCK, James R.; LAMBERT, Douglas M. Becoming a "world class" company with logistics service quality. The International Journal of Logistics Management, v.3, p. 73-80, Janeiro 1992.

7. WARD, James A. Measurement management: what you measure is what you get. Information Systems Management, v. 5, p. 59-61, Winter 1996. 